\title{
Utilização dos serviços odontológicos por crianças de 0 a 9 anos cadastradas em uma Unidade de Saúde da Família de Salvador, Bahia, Brasil
}

\author{
Use of dental services by children from 0 to 9 years registered in a Health Unit of the \\ Family of Salvador, Bahia, Brazil
}

\begin{abstract}
Karina de Andrade Lage ${ }^{1}$, Maria Cristina Teixeira Cangussu ${ }^{2 *}$, Maria Lizzia Moura Ferreira dos Santos ${ }^{3}$, Maria Beatriz Barreto de Sousa Cabral2, Maria Isabel Pereira Vianna², Tatiana Frederico de Almeida²

${ }^{1}$ Especialista em Saúde Pública. Cirurgiã-dentista da Secretaria Municipal de Saúde de Salvador-BA.; ${ }^{2}$ Doutora em Saúde Pública. Professora Adjunta da Universidade Federal da Bahia.; ${ }^{3}$ Doutoranda em Odontologia e Saúde
\end{abstract}

\begin{abstract}
Resumo
Objetivo: o objetivo deste estudo foi verificar a utilização de serviços odontológicos, idade e motivo da primeira consulta odontológica em crianças de 0 a 9 anos cadastradas em uma Unidade de Saúde da Família em Salvador, Bahia, Brasil. Metodologia: realizou-se um estudo descritivo, transversal. Os responsáveis pelas crianças participaram de entrevistas individuais e forneceram informações sobre a idade atual da criança, idade da primeira consulta odontológica, realização de consulta no último ano, tipo de financiamento e motivo da primeira consulta odontológica. As entrevistas foram realizadas durante visitas domiciliares e na respectiva Unidade de Saúde. Os dados foram tabulados e analisados de forma descritiva. Resultados: um total de 151 questionários foram aplicados. Das crianças, 59,6\% nunca realizaram nenhum tipo de consulta odontológica e daqueles que já o tinham feito, $62,30 \%$ foi no último ano e $83,61 \%$ destas realizadas no serviço público. O principal motivo da consulta foi a presença de cárie $(36,07 \%)$. As crianças do sexo feminino realizaram a primeira consulta odontológica em maior proporção que as do sexo masculino, embora esta tenha ocorrido em uma idade mais tardia. Conclusões: Há necessidade de fomentar a utilização do serviço odontológico de forma precoce, permitindo a implementação de políticas públicas de promoção de saúde de forma a garantir o atendimento às crianças desde o primeiro ano de vida. Palavras-chave: Serviços de Saúde. Epidemiologia. Infância.
\end{abstract}

\begin{abstract}
Objective: the aim of this study was to verify the use of dental services, age and reason for the first dental visit in children aged 0 to 9 enrolled in a Family Health Unit in Salvador, Bahia, Brazil. Methods: a descriptive, cross-sectional study was carried out. Those responsible for the children participated in individual interviews and provided information on the child's current age, age at the first dental visit, last year's consultation, type of funding and reason for the first dental visit. The interviews were conducted during home visits and at the respective Health Unit. Data were tabulated and analyzed in a descriptive way. Results: a total of 151 questionnaires were applied. 59.6\% of the children never performed any type of dental consultation and those who had already done it, $62.30 \%$ were in the last year and $83.61 \%$ of these were in the public service. The main reason for the consultation was the presence of caries (36.07\%). The female children performed the first dental visit in a greater proportion than the male ones, although this occurred at a later age. Conclusions: there Is a need to encourage the use of the dental service in an early manner, allowing the implementation of public health promotion policies in order to ensure care for children from the first year of life.

Keywords: Health Services. Epidemiology. Childhood.
\end{abstract}

\section{INTRODUÇÃO}

A odontologia tem avançado na atualidade, superando uma ótica curativa dos problemas bucais para o enfrentamento dos determinantes do processo saúde-doença através da vigilância em saúde (KRAMER et al., 2008). No entanto, apesar da diminuição constatada da cárie dentária na faixa escolar tanto no Brasil, como no mundo, a mesma ainda é a patologia bucal de maior prevalência na infância, afetando com maior frequência e gravidade crianças com condições sócio-econômicas

Correspondente/Corresponding: *Maria Cristina Teixeira Cangussu - End: Rua Araújo Pinho, n. 62, 60 Andar. Faculdade de Odontologia. CEP: 41110-150 Canela. Salvador - BA - Tel: (71) 3283-8990 - E-mail: cangussu@ufba.br desfavoráveis (BALDANI et al., 2017; HAMATI; ROCHA; BALDANI, 2014). Os altos custos de tratamento deste agravo bucal e a possibilidade de utilização de medidas preventivas eficazes, como o uso do fluoreto em larga escala, da educação em saúde e a ampliação do acesso aos serviços públicos de saúde, reforçam a classificação da cárie como um relevante problema de saúde pública (PERES et al., 2003).

Entretanto, em pré-escolares, o controle desta doença ainda é de menor magnitude do que a observada em escolares. Segundo Cangussu et al. (2016) e Melo et al. (2011), o problema ainda se apresenta de forma mais contundente em determinados grupos etários. De acordo com os resultados do SB Brasil 2010 (BRASIL, 2011), aos 5 anos de idade uma criança brasileira possuía em média 
2,43 dentes com experiência de cárie, sendo os piores resultados encontrados nas regiões Norte, Centro-Oeste e Nordeste em comparação ao Sul e Sudeste. Além disso, Baldani et al. (2017) reforçam que para esta faixa etária, ao se comparar os resultados do SB Brasil 2003 com os do SB Brasil 2010, o padrão de falta de acesso aos cuidados com a saúde bucal permaneceu inalterado, com $80 \%$ dos dentes decíduos acometidos por cárie não tratados.

A experiência de cárie dentária durante a infância é causa frequente de dor e má oclusão, tendo como consequênciada perda precoce de dentes decíduos, desconforto na mastigação e fonação que comprometem a qualidade de vida e o desenvolvimento biopsicossocial da criança (HAMATI; ROCHA; BALDANI, 2014; MELO et al., 2011).

A dor de origem dentária é apontada como um significativo problema de saúde pública. As sensações dolorosas são percebidas por todas as idades, e especialmente por crianças (HAMATI; ROCHA; BALDANI, 2014; MELO et al., 2011), e pode interferir em aspectos fundamentais da evolução do crescimento, como na alimentação, no aprendizado e no lazer (ALBUQUERQUE et al., 2016). A presença de dor revelou-se um forte preditor para a consulta odontológica por problemas bucais entre crianças, geralmente decorrente de infecção causada pela cárie dentária ou pela deficiência de tratamentos restauradores ou endodônticos anteriores (ALBUQUERQUE et al., 2016; CAMARGO et al., 2012).

Preconiza-se que a idade ideal para a primeira consulta odontológica é entre 6 e 12 meses de vida, coincidindo com a época da erupção do primeiro dente decíduo (HAMATI; ROCHA; BALDANI, 2014; KRAMER et al., 2008). Entretanto, de acordo com Edwards (2015), a idade média da primeira consulta odontológica nos EUA ainda é de 2,5 anos de idade. Os resultados da investigação deste autor indicaram que $3 / 4$ dos pais não estão obedecendo a orientação da American Academy of Pediatric Dentistry (AAPD) para levarem seus filhos ao dentista com 01 ano de idade.

A assistência odontológica ofertada em serviços públicos no Brasil não tem se mostrado capaz de atender a grande demanda de pacientes, gerando dessa forma, poucas oportunidades para a prática de prevenção, diagnóstico e tratamento precoce, tanto da cárie como de outras patologias bucais (ALBUQUERQUE et al., 2016; HAMATI; ROCHA; BALDANI, 2014). Em um estudo realizado por Kramer et al. (2008) no município de Canela, RS, apenas $13,3 \%$ das crianças de 0 a 5 anos haviam realizado algum tipo de consulta odontológica. Segundo Hamati, Rocha e Baldani (2014) a Pesquisa Nacional por Amostra de Domicílio (PNAD) de 2008, mostrou que 77,9\% das crianças brasileiras entre 0 e 4 anos de idade nunca haviam realizado algum tipo de consulta odontológica.

Segundo Cangussu et al. (2016), as principais dificuldades para se organizar a atenção à saúde bucal na primeira infância no Brasil são o modelo primordialmente cirúrgico-restaurador e direcionado ao escolar, a pequena importância concedida à dentição decídua, a dificuldade no manejo de crianças de pequena idade, a dieta e o consumo de açúcar neste período, além de hábitos de aleitamento e higiene bucal. Até a criação do Sistema Único de Saúde (SUS), as Políticas de Saúde Bucal privilegiavam as crianças em idade escolar (6 a 14 anos) na oferta de assistência odontológica programática. Aos demais grupos populacionais, restava o atendimento da urgência de origem odontológica. Porém, ainda hoje, os serviços odontológicos concentram pouca atenção à faixa pré-escolar (CAMARGO et al., 2012).

De acordo com Camargo et al. (2012), estudos sobre o uso dos serviços odontológicos entre pré-escolares são exíguos e em grande maioria advêm de países desenvolvidos. Dentre os países classificados como de baixa e média renda, foram encontradas análises em populações do Brasil, México e Filipinas. Nesses países, estudos de coorte de nascimento com foco em problemas de saúde bucal são ainda mais raros (PERES et al., 2010). Para Kramer et al. (2008) essas pesquisas são significativas, pois possibilitam, a partir de uma necessidade verificada, a construção de políticas públicas de promoção de saúde com o objetivo de esclarecer e conduzir a população ao atendimento odontológico precoce.

Segundo Baldani et al. (2017) uma grande gama de variáveis contribui para facilitar ou restringir o acesso e uso dos serviços de saúde, e esses fatores foram integrados em um modelo explicativo proposto por Andersen. O modelo comportamental de Andersen relata que o uso de serviços de saúde resulta da interação de alguns fatores individuais, características do sistema de saúde, contexto social e experiências passadas dos indivíduos em acessar os serviços. Portanto, o uso dos serviços de saúde estaria relacionado a uma predisposição individual diretamente influenciada por características sociodemográficas, preferências, expectativas em relação à saúde e conhecimento sobre os serviços, o nível de necessidade ligado a autopercepção, gravidade da doença, limitações da atividade e a presença de fatores que permitem o uso de serviços de saúde, como renda, suporte social e fonte regular de cuidados.

Assim, o objetivo deste trabalho foi verificar a utilização dos serviços odontológicos pelas crianças de 0 a 9 anos cadastradas em uma Unidade de Saúde da Família de Salvador - BA no ano de 2018, assim como identificar a média de idade da primeira consulta e variáveis relacionadas à mesma.

\section{METODOLOGIA}

Foi desenvolvido um estudo transversal do qual participaram todos os responsáveis por crianças de 0 a 9 anos cadastradas em uma equipe de uma Unidade de Saúde da Família de Salvador - BA, sendo a população adscrita da equipe composta por 11.842 indivíduos de baixo nível sócio econômico.

Para a obtenção dos dados foram necessárias algumas fontes de informações: o cadastro das famílias na 
unidade para identificar o total de crianças de 0-9 anos cadastradas na área de abrangência. Foram considerados elegíveis todos os domicílios com crianças menores de 10 anos de idade.

Foi realizada entrevista com os responsáveis pelas crianças a partir de um instrumento de pesquisa com questões objetivas, aplicado pela equipe de saúde bucal (cirurgiã dentista e auxiliar de saúde bucal), 3 agentes comunitários de saúde, enfermeira e médico da Unidade de Saúde da Família (7 pessoas ao total), durante visitas domiciliares, consultas de puericultura e pediatria. Foram avaliados os seguintes itens: idade em que se deu a primeira consulta odontológica da criança; caso ela já tivesse ocorrido, se foi utilizado serviço público ou privado, qual foi o motivo da consulta (dor, cárie, sangramento gengival, trauma/queda, orientação de higiene bucal, solicitação médico/enfermeira e a opção outros) e se a criança compareceu ao dentista no último ano. Realizou-se um treinamento com os profissionais previamente à aplicação do instrumento, com duração de 4 horas. Durante uma reunião de equipe, o mesmo foi discutido item a item com o objetivo de uniformizar sua utilização e posteriormente ele foi testado em 5 famílias da área de abrangência de outra equipe da unidade para ajustes.

Os dados foram tabulados em planilhas utilizando o programa Excel 2010. Os resultados foram analisados descritivamente, sendo apresentados por meio de gráficos e tabelas. Para a comparação das médias utilizou-se o teste $t$ de Student, e para as proporções o qui-quadrado, ambos com o nível de confiança de $95 \%$.

O protocolo desta pesquisa foi aprovado pelo Comitê de Ética em Pesquisa da Faculdade de Odontologia da UFBA, sob o CAAE 78351317.0.0000.5024.

\section{RESULTADOS}

Participaram das entrevistas 151 responsáveis, o que compreende uma taxa de resposta de $100 \%$. Não houve recusa em participar do estudo. A média de idade da população de crianças foi de 4,4 anos ( $D P=2,8)$. Destas, $59,6 \%$ nunca havia realizado nenhum tipo de consulta odontológica.

A tabela 1 apresenta algumas condições da população de estudo. A distribuição percentual por sexo apresentou uma maioria de crianças do sexo masculino $(53,64 \%)$. Quanto à distribuição das crianças por idade, houve um equilíbrio entre o número de indivíduos das idades avaliadas, exceto para a idade de 8 anos, que apresentou um percentual menor (4\%).

Dentre as crianças que já tinham realizado consulta odontológica anterior ( $n=61 ; 40,4 \%), 62,30 \%$ o fizeram no último ano. A maioria das consultas $(83,61 \%)$ ocorreu no serviço público e apenas $12,39 \%$ das consultas foram realizadas no serviço privado.

O principal motivo da consulta relatado pelos entrevistados foi a presença de cárie na cavidade bucal do menor (36,07\%). Os itens "necessidade de orientação de higiene bucal" e "solicitação da consulta por profissional médico ou enfermeira" apresentaram o mesmo percentual de $16,39 \%$, seguidos pelos itens "outros motivos" $(13,11 \%)$, "trauma ou queda" $(9,84 \%)$ e "dor" $(8,20 \%)$. O motivo "sangramento gengival" não foi citado por nenhum dos entrevistados.

Tabela 1 - Distribuição percentual das crianças por sexo, idade, consulta no último ano, tipo de serviço utilizado e motivo da consulta, Salvador - BA, 2018. ( $n=151)$.

\begin{tabular}{lrr}
\hline Variáveis & $\mathrm{n}$ & $\%$ \\
\hline Sexo & & \\
Masculino & 81 & 53,64 \\
Feminino & 70 & 46,64 \\
Idade (em anos) & & \\
$0-1$ & 28 & 18,5 \\
2 & 18 & 11,9 \\
3 & 18 & 11,9 \\
4 & 17 & 11,2 \\
5 & 16 & 10,6 \\
6 & 13 & 8,7 \\
7 & 16 & 10,6 \\
8 & 6 & 4,0 \\
9 & 19 & 12,6 \\
Utilização do serviço odontológico & & \\
Sim & 61 & 40,4 \\
Não & 90 & 59,6 \\
Utilização do serviço odontológico no último ano* & & \\
Sim & 38 & 62,3 \\
Não & 23 & 37,7 \\
Tipo de financiamento do serviço utilizado & & \\
Público & 51 & 83,6 \\
Privado & 10 & 16,4 \\
Motivo da utilização do serviço odontológico & & \\
Dor & 5 & 8,2 \\
Cárie & 22 & 36,0 \\
Sangramento gengival & - & -- \\
Trauma/ queda & 6 & 9,8 \\
Orientação higiene oral & 10 & 16,4 \\
Solicitação médico/ enfermeira & 10 & 16,4 \\
Outros & 8 & 13,1 \\
\hline
\end{tabular}

Fonte: Dados da pesquisa.

Quando foram analisados os fatores associados à primeira consulta odontológica, o sexo masculino apresentou uma média de idade inferior ao sexo feminino, estatisticamente significante. A idade média da primeira consulta odontológica para o sexo masculino foi de 2,93 $(2,25-3,62$ IC $95 \%)$ anos, enquanto para o sexo feminino foi de 4,03 (3,05-5,01 IC 95\%) anos ( $p$ valor $=0,047)$.

A Tabela 2 apresenta as diferenças na distribuição da realização da consulta odontológica no último ano e em períodos anteriores. Observou-se que um maior número de indivíduos do sexo feminino foi à consulta no último ano. Quanto a utilização de serviço público ou privado para a consulta odontológica não houve diferença significativa entre os sexos em ambos os tipos de serviço. 
Tabela 2 - Distribuição percentual por sexo da utilização do serviço odontológico no último ano, em anos anteriores e de indivíduos que nunca foram ao serviço odontológico, Salvador - BA, 2018.

\begin{tabular}{|c|c|c|c|c|c|c|c|}
\hline Variáveis & $\begin{array}{c}\text { Utilizaram serviço } \\
\text { odontológico no último ano }\end{array}$ & & $\begin{array}{l}\text { Não utilizaram serviço } \\
\text { odontológico no último } \\
\text { ano }\end{array}$ & & $\begin{array}{l}\text { Nunca utilizaram serviço } \\
\text { odontológico }\end{array}$ & & $P$ valor \\
\hline & $\mathrm{n}$ & $\%$ & $\mathrm{n}$ & $\%$ & $\mathrm{n}$ & $\%$ & \\
\hline \multicolumn{8}{|l|}{ Sexo } \\
\hline Masculino & 18 & 56,3 & 14 & 43,7 & 49 & - & 0,30 \\
\hline Feminino & 20 & 69,0 & 9 & 31,0 & 41 & & \\
\hline \multicolumn{8}{|l|}{ Tipo de serviço } \\
\hline Público & 31 & 60,8 & 20 & 39,2 & - & - & 0,58 \\
\hline Privado & 7 & 70,0 & 3 & 30,0 & - & - & \\
\hline $\begin{array}{l}\text { Nunca utilizaram } \\
\text { serviço odontológico }\end{array}$ & & & & & 90 & & \\
\hline
\end{tabular}

Fonte: Dados da pesquisa.

\section{DISCUSSÃO}

Os resultados mostraram que apenas $40,4 \%$ das crianças pesquisadas já realizaram algum tipo de consulta odontológica. Esse resultado apresentou-se mais favorável do que o encontrado no estudo realizado no município de Canela no ano de 2008, em que apenas $13,3 \%$ das crianças de 0 a 5 anos já haviam ido ao dentista. (KRAMER et al., 2008) No entanto, nesse mesmo estudo de Canela, se considerarmos apenas as idades de 4 e 5 anos, essa média eleva-se para $26,5 \%$ dos pré-escolares. É importante ressaltar que este trabalho referiu-se as idades de 0-9 anos, com o objetivo de identificar a atenção à saúde bucal na infância. Entretanto, a grande maioria dos trabalhos na literatura refere-se apenas aos pré - escolares como população de estudo.

Segundo Krammer et al. (2008), estudos como este realizado em uma Unidade de Saúde da Família na cidade de Salvador podem auxiliar na implementação de políticas públicas de saúde dirigidas ao incentivo da oferta de atendimento odontológico para indivíduos em idade precoce e de campanhas que incentivem sua utilização por parte da população. Segundo Miranda e Peres (2013, apud Andersen e Davidson, 2000) a utilização de serviços de saúde é a comprovação do acesso realizado, sua averiguação possibilita conhecer a efetividade destes serviços $\mathrm{e}$, consequentemente, a presença ou não de justiça social.

Na pesquisa realizada por Noro et al. (2008) com crianças de 5 a 9 anos no município de Sobral, Ceará, $49,1 \%$ delas nunca tiveram acesso a tratamento dentário, enquanto pouco mais da metade $(50,9 \%)$ já realizaram este tipo de tratamento em algum momento de sua vida. Na investigação de Hamati, Rocha e Baldani (2014) no município de Ponta Grossa, Paraná, $42 \%$ das crianças de 3 a 5 anos jamais foram ao dentista. Esses resultados se aproximam dos encontrados no presente estudo, onde $59,6 \%$ dos avaliados nunca realizaram consulta odontológica, e $40,4 \%$ já realizaram esse tipo de consulta (tabela 1 ). Noro et al. (2008), que utilizaram faixa etária similar a deste trabalho, afirmaram que analisar a utilização de serviços odontológicos e os fatores relacionados a ela é condição fundamental para perceber o efetivo alcance do desafio da universalização do acesso à saúde proposto pelo SUS.
Quanto a idade para a realização da primeira consulta odontológica, a média desta idade em ambos os sexos neste estudo foi maior que a preconizada pela literatura, que afirma que a primeira consulta odontológica deve coincidir com a erupção dos primeiros dentes decíduos, entre 6 e 12 meses de idade. Para os indivíduos do sexo masculino, a média de idade da primeira consulta foi de 2,93 anos enquanto para os do sexo feminino foi de 4,03 anos. Isso reflete ainda o desconhecimento dos pais sobre este procedimento, a pouca importância dada aos dentes decíduos, a dificuldade de acesso e as dificuldades inerentes ao tratamento odontológico em crianças. A realização da primeira consulta odontológica de maneira precoce ainda não é vista pela sociedade como uma maneira eficiente de reduzir a prevalência das doenças bucais e, consequentemente, reduzir os custos do tratamento de suas consequências.

Nesta investigação foi observado que um maior número de indivíduos do sexo masculino nunca havia realizado consulta odontológica $(54,44 \%)$ quando comparados com os do sexo feminino $(45,56 \%)$ (tabela 2$)$. $O$ fato de indivíduos do sexo feminino terem uma maior preocupação com a sua saúde tem sido relatado na literatura independentemente da faixa etária. Segundo Pinheiro et al. (2002), estudos sobre diferenças de gênero na saúde em sociedades industrializadas demonstram que as muIheres, apesar de viverem mais que os homens, relatam mais morbidade e problemas psicológicos e utilizam mais serviços de saúde.

É importante lembrar que entre os indivíduos da faixa etária estudada a procura por atendimento odontológico está condicionada a decisões de seus responsáveis. Para Camargo et al. (2012), durante a infância é principalmente a mãe quem pontua a necessidade de levar a criança aos serviços de saúde. Estudo realizado por Almeida et al. (2012) associou problemas mentais maternos ao desenvolvimento de cárie em pré-escolares, salientando que o cuidado materno com seus filhos pode ser afetado pelos agravos mentais.

Outro resultado que chama a atenção neste estudo é o percentual das consultas odontológicas desta população no serviço público $(83,61 \%)$. Este fato pode ser relacio- 
nado à pesquisa ter sido desenvolvida em um território onde a população apresenta situação sócio-econômica desfavorável e contar com uma Unidade de Saúde da Família em seu território, o que de certa forma assegura maior facilidade ao acesso. Este resultado coincidiu com a investigação realizada por Noro et al. (2008) na qual $85,4 \%$ das crianças que tiveram acesso ao tratamento odontológico o realizou na rede pública, ou seja, por intermédio do SUS.

Das crianças que já realizaram consulta odontológica, $37,7 \%$ delas não estiveram no dentista no último ano, demonstrando que o acesso não tem se dado de forma regular (tabela 1). Este achado também se apresentou próximo ao encontrado no município de Sobral no estudo realizado por Noro et al. (2008), no qual $34,7 \%$ das crianças que havia tido acesso ao tratamento dentário o realizaram há mais de um ano. No entanto, na pesquisa de Hamati, Rocha e Baldani (2014), 67,4\% das crianças de 3 a 5 anos de Ponta Grossa, Paraná, não havia realizado consulta há um ano ou menos. Assumindo que esta faixa etária é inferior àquela deste trabalho, é factível a identificação de maior ausência de serviços.

Quanto ao motivo da primeira consulta odontológica, a presença de cárie foi a razão relatada por 36,09\% dos participantes, demonstrando como esta doença ainda é prevalente na faixa etária avaliada. Estudo realizado por Almeida et al. (2009) encontrou em crianças de 0 a 5 anos residentes em áreas de abrangência do Programa Saúde da Família, na cidade de Salvador, uma taxa de prevalência de cárie de $49,6 \%$. Nesta investigação, se o percentual de crianças que foi ao dentista devido à cárie for somado ao percentual devido à dor, que geralmente em crianças está relacionada à presença de cárie, tem-se quase metade das consultas odontológicas determinadas pela presença desta doença na cavidade bucal.

No entanto, o motivo da primeira consulta odontológica em busca de orientação de higiene oral foi mencionado por $16,39 \%$ dos entrevistados neste estudo, demonstrando certa preocupação por parte dos responsáveis na prevenção das patologias bucais. Camargo et al. (2012) associaram positivamente consultas de rotina em crianças com alguns comportamentos maternos, como a realização de pelo menos sete consultas de pré-natal, utilização adequada de puericultura e uso regular de serviços odontológicos. Além disso, pelo fato desta pesquisa ser realizada com uma população cadastrada em uma Unidade de Saúde da Família, onde há valorização e preocupação com o trabalho em equipe, 16,39\% dos responsáveis relataram a razão "solicitação médico/ enfermeira" para a realização da consulta odontológica.

Segundo Pinheiro e Torres (2006), não apenas a oferta de serviços odontológicos de qualidade é um fator importante para a ampliação do acesso da população a serviços resolutivos, como também se faz necessária a percepção das necessidades de saúde bucal por parte dos indivíduos, para que ocorra a procura por estes serviços. Para estes autores, políticas educativas que ampliem o conhecimento da população sobre medidas preventivas e orientação na busca por cuidados de saúde são questões importantes para a gestão em saúde em qualquer país.

O motivo "trauma/queda" para a consulta odontológica foi observado em 9,84\% dos entrevistados. A ocorrência de trauma na dentição decídua é comum. Kramer et al. (2009) em um estudo desenvolvido no município de Canela, Rio Grande do Sul, encontraram uma prevalência de $23,6 \%$ desse tipo de traumatismo, sem diferença significativa entre os sexos. A faixa etária mais acometida foi entre dois e três anos, e o dente mais atingido foi o incisivo central superior (83,8\%).

A não citação dos entrevistados do motivo "sangramento gengival" para consulta odontológica pode estar refletindo um desconhecimento dos pais sobre as patologias periodontais, a utilização de técnica de escovação incorreta ou mesmo a maior ocorrência da cárie na dentição decídua, o que faz com que outras patologias permaneçam em segundo plano. Para Almeida et al. (2009) são poucas as informações epidemiológicas sobre alterações periodontais em crianças na faixa etária pré-escolar no Brasil, trazendo dificuldade em se comparar os resultados.

Por fim, se faz importante enfatizar que os achados do presente estudo devem ser analisados com ponderação devido ao pequeno tamanho da população pesquisada, ao processo de seleção da mesma e as limitações metodológicas inerentes ao processo de amostragem, que não permite estimativas para a população de Salvador nessa faixa etária. Além disso, não foi possível restringir a população a apenas pré - escolares por este limite do tamanho da população. Bem como a necessidade real da utilização dos dados para organização dos serviços de saúde da USF. O fato do estudo também não ter abrangido todas as áreas do município em questão é um fator a ser considerado. Todavia, há de se ressaltar a importância do emprego da metodologia epidemiológica para o reconhecimento da realidade local do serviço de saúde, o que visa o aprimoramento de sua organização e efetividade com a perspectiva de um cuidado mais integral.

\section{CONCLUSÕES}

A utilização de serviços odontológicos por crianças nesta unidade de saúde ainda é considerada baixa (40,4\%);

- a procura por serviços odontológicos é tardia - (2,94 anos para o sexo masculino e 4,04 anos para o sexo feminino), com maior utilização para o sexo feminino $(54,44 \%)$, no serviço público $(83,61 \%)$;

- o principal motivo para a primeira consulta é a presença da cárie dentária (36,09\%).

\section{REFERÊNCIAS}

ALBUQUERQUE, Y. E. et al. Perfil do atendimento odontológico no Serviço de Urgência para crianças e adolescentes da Faculdade de Odontologia de Araraquara UNESP. Rev. Odontol. UNESP, Marília, v. 45, n. 2, p. 115-120, 2016. 
ALMEIDA, T. F. et al. Condições de saúde bucal de crianças na faixa etária pré-escolar, residentes em áreas de abrangência do Programa Saúde da Família em Salvador, Bahia, Brasil. Rev. Bras. Saúde Matern. Infant., Recife, v. 9, n. 3, p. 247-252, 2009.

ALMEIDA, T. F. et al. Family context and incidence of dental caries in preschool children living in areas covered by the Family Health Strategy in Salvador, Bahia State, Brazil. Cad. Saúde Pública, Rio de Janeiro, v. 28, n. 6, p. 1183-1195, 2012.

BALDANI, M. H. et al. Assessing the role of appropriate primary health care on the use of dental services by Brazilian low-income preschool children. Cad. Saúde Pública, Rio de Janeiro, v. 33, n. 11, p. e00158116, 2017.

BRASIL. Ministério da Saúde. Secretaria de Atenção à Saúde. Departamento de Atenção Básica. Projeto SB Brasil 2010: condições de saúde bucal da população brasileira 2010: resultados principais. Ministério da Saúde, Secretaria de Atenção à Saúde, Departamento de Atenção Básica. Brasília: Ministério da Saúde, 2011.

CAMARGO, M. B. J. et al. Preditores da realização de consultas odontológicas de rotina e por problema em pré-escolares. Rev. Saúde Pública, São Paulo, v. 46, n. 1, p. 87-97, 2012.

CANGUSSU, M. C. T. et al. Fatores de risco para a cárie dental em crianças na primeira infância, Salvador - BA. Rev. Bras. Saúde Matern. Infant., Recife, v. 6, n. 3, p. 309-317, 2016.

EDWARDS, T. Pesquisa descobre que primeira visita dental infantil não cumpre a diretriz da AAPD. 2015. Disponível em: <http://www. drbicuspid.com/ index.aspxsec=sup\&sub=hyg\&pag=dis\&ltemID=3173 29\&wf=2141>. Acesso em: 01 fev. 2018.

HAMATI, F.; ROCHA, J. S.; BALDANI, M. H. Prevalência de cárie, dor e uso de serviços odontológicos por crianças em áreas com e sem Equipes de Saúde Bucal na Estratégia Saúde da Família no município de Ponta Grossa, Paraná, Brasil. Rev. Bras. Pesq. Saúde, Vitória, v. 16, n. 3, p. 48-57, 2014.
KRAMER, P. F. et al. Utilização dos serviços odontológicos por crianças de 0 a 5 anos de idade no município de Canela, Rio Grande do Sul, Brasil. Cad. Saúde Pública, Rio de Janeiro, v. 24, n. 1, p. 150-156, 2008.

KRAMER, P. F. et al. Traumatismo na Dentição Decídua e Fatores Associados em Pré-Escolares do Município de Canela/RS. Pesq. Bras. Odontop. Clín. Integr., João Pessoa, v. 9, n. 1, p. 95-100, 2009.

MELO, M. M. D. C. et al. Fatores associados à cárie dentária em pré-escolares do Recife, Pernambuco, Brasil. Cad. Saúde Pública, Rio de Janeiro, v. 27, n. 3, p. 471-485, 2011.

MIRANDA, C. B.; PERES, M. A. Determinantes da utilização de serviços odontológicos entre adultos: um estudo de base populacional em Florianópolis, Santa Catarina, Brasil. Cad. Saúde Pública, Rio de Janeiro, v. 29, n. 11, p. 2319-2332, 2013.

NORO, R. A. et al. A utilização de serviços odontológicos entre crianças e fatores associados em Sobral, Ceará, Brasil. Cad. Saúde Pública, Rio de Janeiro, v. 24, n. 7, p. 1509-1516, 2008.

PERES, M. A. et al. Determinantes sociais e biológicos da cárie dentária em crianças de 6 anos de idade: um estudo transversal aninhado numa coorte de nascidos vivos no Sul do Brasil. Rev. Bras. Epidemiol., São Paulo, v. 6, n. 4, p. 293-306, 2003.

PERES, M. A. et al. Oral health follow-up studies in the 1993 Pelotas (Brazil) birthcohortstudy: methodologyand principal results. Cad. Saúde Pública, Rio de Janeiro, v. 26, n. 10, p. 1990-1999, 2010.

PINHEIRO, R. S. et al. Gênero, morbidade, acesso e utilização de serviços de saúde no Brasil. Ciênc. Saúde Colet., Rio de Janeiro, v. 7, n. 4, p. 687-707, 2002.

PINHEIRO, R. S.; TORRES, Z. G. Uso de serviços odontológicos entre os Estados do Brasil. Ciênc. Saúde Colet., Rio de Janeiro, v. 11, n. 4, p. 999-1010, 2006.

Submetido em: $21 / 02 / 2019$

Aceito em: 23/09/2019 